\title{
A Machine Learning Approach for Product Matching and Categorization
}

\author{
Use case: Enriching Product Ads with Semantic Structured Data
}

\author{
Petar Ristoski $^{a}$, Petar Petrovski ${ }^{a}$, Peter Mika $^{\mathrm{b}}$, Heiko Paulheim ${ }^{\mathrm{a}}$, \\ ${ }^{a}$ Data and Web Science Group, University of Mannheim, B6, 26, 68159 Mannheim \\ E-mail: petar.ristoski@informatik.uni-mannheim.de,petar@informatik.uni-mannheim.de, \\ heiko@informatik.uni-mannheim.de \\ b Yahoo Labs, London, UK \\ E-mail: pmika@yahoo-inc.com
}

\begin{abstract}
Consumers today have the option to purchase products from thousands of e-shops. However, the completeness of the product specifications and the taxonomies used for organizing the products differ across different e-shops. To improve the consumer experience, approaches for product integration on the Web are needed. In this paper, we present an approach that leverages deep learning techniques in combination with standard classification approaches for product matching and categorization. In our approach we use structured product data as supervision for training feature extraction models able to extract attribute-value pairs from textual product descriptions. To minimize the need for lots of data for supervision, we use neural language models to produce word embeddings from large quantities of publicly available product data marked up with Microdata, which boost the performance of the feature extraction model, thus leading to better product matching and categorization performances. Furthermore, we use a deep Convolutional Neural Network to produce image embeddings from product images, which further improve the results on both tasks.
\end{abstract}

Keywords: Microdata, schema.org, Data Integration, Product Data, Deep Machine-Learning

\section{Introduction}

In the recent years, with the advancements of the Internet and e-shops services, the amount of products sold through e-shops has grown rapidly. A recent study estimates that the total e-commerce retail sales for the fourth quarter of 2015 in the USA only were 89.1 billion dollars [3]. However, there is one big issue in the process of product search and purchase that consumers have to deal with. The same product may be found on many different e-shops, but the information about the products offers greatly differ across different e-shops. Furthermore, there are no global identifiers for products, and offers are most often not interlinked among each other. Therefore, there is no simple way for the consumers to find all the necessary information and best prices for the products they search for. To offer a better user experience, there are many products aggregators, like Google Product Search ${ }^{1}$, PriceGrabber ${ }^{2}$, and Shopzila ${ }^{3}$, trying to integrate and categorize products from many e-shops and many different merchants. However, the task for identifying the same product offers across thousands of e-shops and integrating the information into a single representation is not trivial. Furthermore, to allow the users better navigation and product search, the product aggregators have to deal with the task of organizing all the products according to a product taxonomy.

To support the integration process, e-shops are increasingly adopting semantic markup languages such

\footnotetext{
${ }^{1}$ http://www.google.com/shopping

${ }^{2}$ http: //www.pricegrabber.com/

${ }^{3}$ http://www. shopzilla.com/
} 
as Microformats, RDFa and Microdata, to annotate their content, making large amounts of product description data publicly available. In this paper, we present an approach that leverages deep learning techniques in combination with standard classification approaches for product matching and categorization from HTML annotations. We focus on data annotated with the Microdata markup format using the schema.org vocabulary. Recent works $[19,20]$ have shown that the Microdata format is the most commonly used markup format, with highest domain and entity coverage. Also, schema.org is the most frequently used vocabulary to describe products. Although considered structured annotations, empiric studies have shown that the vast majority of products are annotated only with a name and a textual description. This helps identifying the relevant parts on the Website, but leads to rather shallow textual data, which has to be tackled with methods for unstructured data.

In a previous work [26] we proposed an approach for enriching structured product ads with data extracted from HTML pages that contain semantic annotations. The approach is able to identify matching products in unstructured product descriptions using the database of structured product ads as supervision. We identified the Microdata dataset as a valuable source for enriching existing structured product ads with new attributes.

In this paper we enhance the existing approach using deep learning techniques. First, we show that using neural text embeddings from large quantities of Microdata products data can significantly increase the performance of a Conditional Random Field model for attribute-value pair extraction from products descriptions. Then, we use a deep Convolutional Neural Network to produce image embeddings from product images, which can be used as a weak indicator for the task of product matching, and as a strong indicator for the task of product classification. We show that neural document embeddings outperform baseline approaches for products matching and produce comparable results with supervised approaches for product classification. Finally, we use the models in the use case of enriching product ads with metadata from HTML annotations.

The rest of this paper is structured as follows. In Section 2, we give an overview of related work. In Section 3 and Section 4, we introduce our methodology for product matching and categorization, respectivly. In Section 5, we present the results of matching unstructured product descriptions, followed by the evalu- ation of the product ads enrichment with metadata extracted from HTML annotations in Section 6. In Section 7 we present the results of the product categorization approach. We conclude with a summary and an outlook on future work.

\section{Related Work}

Both product matching and product categorization for the Web have been explored with various approaches and methods within the last years.

\subsection{Product matching}

Since there are no global identifiers for products, and links between different e-commerce Web pages are also scarce, finding out whether two offers on different Web pages are referring to the same product is a non-trivial task. Therefore, product matching deals with identifying pairs or sets of identical products.

Ghani et al. [5] first presented enriched product databases with attribute-value pairs extracted from product descriptions on the Web, by using Naive Bayes in combination with a semi-supervised co-EM algorithm to extract attribute-value pairs from text. An evaluation on apparel products shows promising results, however the system is able to extract attributevalue pairs only if both the attribute and the value appear in the text.

The XploreProducts.com platform detailed in [28] integrates products from different e-shops annotated using RDFa annotations. The approach is based on several string similarity functions for product matching. The approach is extended by using a hybrid similarity method and hierarchical clustering for matching products from multiple e-shops [1].

Kannan et al. [9] use the Bing products catalog to build a dictionary-based feature extraction model. Later, the features of the products are used to train a Logistic Regression model for matching product offers to the Bing shopping data. Another machine learning approach for matching products data is proposed in [12]. First, several features are extracted from the title and the description of the products using manually written regular expressions. In contrast, named entity recognition based feature extraction models are developed in [18] and [26]. Both approaches use a CRF model for feature extraction, however [18] has a limited ability to extract explicit attribute-value pairs, which is improved upon in [26]. 
The first approach to perform products matching on Microdata annotations is presented in [24], based on the Silk rule learning framework [8]. To do so, different combination of features (e.g. bag of words, dictionary-based, regular expressions etc.) from the product descriptions are used. The work has been extended in [25], where the authors developed a genetic algorithm for learning regular expressions for extracting attribute-value pairs from products.

The authors of [23] perform product matching on a dataset of the Bing search engine. In their approach, the authors use historical knowledge to generate the attributes and to perform schema matching. In particular, they visit the merchant web page to compare the values of the products in the catalog with the values on the web page, converting the problem to a standard table schema matching problem. Next, the authors use instance-based schema matching to align the attributes' names in the catalog to the ones on the merchant web page.

In [6] the authors propose an unsupervised webbased enrichment approach for the purpose of product matching. They start with enriching the title of the product with tokens retrieved using a web search engine, i.e., they use the title of the product as a query to a search engine, and the top K returned tokens are used for the enrichment. To identify the relevance of each token in the title, they again use web search, i.e., the token that returns more results is more relevant for the given product. The pairwise product matching is performed based on cosine similarity, using prefix filtering techniques as a blocking approach.

A similar approach for enriching product descriptions with tokens using web search engine is proposed in [16]. The authors propose a similar approach to [6]. The approach first enriches the offer's title with tokens using web search engine. Then, it uses Community Detection for an approximate matching approach which is responsible for computing the distance between the pairs, computed based on the strength of the constructed "social" network between the private tokens. The approach is evaluated on the Abt-Buy dataset ${ }^{4}$ and a small custom dataset.

While the discussed studies above have implemented diverse approaches (classifiers, genetic programming, string similarities), the feature extraction techniques used are mostly dependent on a supervised

\footnotetext{
${ }^{4}$ http://dbs.uni-leipzig.de/en/research/projects/ object $\backslash$ matching/fever/benchmark $\backslash \backslash \backslash$ _dataset $\backslash \backslash$ for _entity $\backslash$ resolution
}

dictionary-based approach. Recently, a handful of approaches employed word embeddings for getting features from product data, however none are concerned with the problems of classification and/or matching. The approach by Grbovic et al. [7] discusses the problem of product recommendations as a part of online advertisements. To perform recommendations, the authors use word2vec [22] to create product embeddings from product titles for product-to-product predictions, as well as paragraph2vec [14] to create user embeddings for user-to-products predictions. Similarly, in [30], the authors present a product recommendation system for microblogging websites where the main idea is that users and products can be represented in the same feature space by employing word $2 \mathrm{vec}$ to calculate the feature vectors.

In [27], the authors present a system that automatically estimates the quality of machine translated segments of product offers. The authors again use word embeddings, specifically paragraph2vec [14], to learn feature vectors from the product title. These vectors are then used to that predict post-edition effort (HTER) on products from three different categories.

To the best of our knowledge, product matching based on image features has only been applied in the apparel domain [11]. As apparel product characteristics are primarily visual, image matching is less demanding compared to other products, for example, electronics. Similarly, with the rapid development of deep learning and neural nets for image processing, there are several approaches to recommend clothing based on images [17].

\subsection{Product classification}

Product classification deals with assigning a set of labels from a product hierarchy to a product. Since not all web sites use a hierarchy, and those who use one are unlikely to use the same, a unified classification of products from different web sites is needed to provide the user with useful browsing and searching functionalities.

While there are several approaches concerned with product data categorization [23,9,24,28], the approach by Meusel at el. [20] is the most recent approach for exploiting Microdata annotations for categorization of product data. In that approach, the authors exploit the already assigned $\mathrm{s}$ : Category $\mathrm{y}^{5}$ property to develop

\footnotetext{
${ }^{5}$ In this paper, $\mathrm{s}$ is used as a shorthand notation for the htt $\mathrm{p}: / / \mathrm{schema}$. org/ vocabulary
} 
distantly supervised approaches to map the products to set of target categories from an existing product cata$\log$.

Although there are a lot of approaches for products matching and classification based on text features, only a few are using image features for the given task. Kannan et al. [10] proposed one of the first approaches for product categorization that besides text features uses image features. The approach is based on Confusion Driven Probabilistic Fusion++, which is cognizant of the disparity in the discriminative power of different types of signals and hence makes use of the confusion matrix of dominant signal (text) to prudently leverage the weaker signal (image), for an improved performance. In our paper, we follow the same setup for building the classifier, however, we use a different image feature extraction technique, i.e., we use deep neural nets image embeddings, while they use simple spatial image features.

\section{Product Matching Approach}

In our approach, we use different feature extraction methods to derive a set of useful features for the product matching task.

\subsection{Problem Statement}

We have a database $A$ of structured products and a dataset of unstructured product descriptions $P$ extracted from the Web. Every record $a \in A$ consists of a title, description, URL, and a set of attribute-value pairs extracted from the title of the product, where the attributes are numeric, categorical, or free-text. Every record $p \in P$ consists of a title and a description as unstructured textual fields, and a product image. Our objective is to use the structured information from the product set $A$ as supervision for identifying duplicate records in $P$, in combination with neural text embeddings extracted from all the records in $P$. More precisely, we use the structured information as a supervision for building a feature extraction model able to extract attribute-value pairs from the unstructured product descriptions in $P$. After the feature extraction model is applied, each product $p \in P$ is represented as a vector of attributes $F_{p}=\left\{f_{1}, f_{2}, \ldots, f_{n}\right\}$, where the attributes are numerical or categorical. Then we use the attribute vectors to build a machine learning model able to identify matching products. To train the model we manually label a small training set of matching and non-matching unstructured product offers.

\subsection{Methodology}

Our approach for products matching consists of three main steps: (i) feature extraction, (ii) calculating similarity feature vectors and (iii) classification. The overall design of our system is illustrated in Fig. 1. The workflow runs in two phases: training and application. The training phase starts with preprocessing both the structured and the unstructured Web product descriptions. Then, we build four feature extraction models as follows:

Dictionary-Based We build a dictionary of the product attributes and their values present in the structured product descriptions.).

Conditional Random Field (CRF) We build a CRF model with a set of discrete features.)

CRF with Text Embeddings In order to handle the dynamic text patterns in product descriptions we enhance the training of the preceding CRF model with text embedding features. This approach is detailed in Section 3.3.2).

Image Feature Extraction Model In addition to the textual features, we furthermore build an image embeddings model.

The approaches are detailed in section 3.3.

Next, we manually label a small training set of matching and non-matching unstructured pairs of product descriptions. Subsequently, we calculate the similarity feature vectors for the labeled training product pairs (Section 3.4). In the final step, the similarity feature vectors are used to train a classification model (Section 3.5). After the training phase is over, we have a trained feature extraction model and a classification model.

In the application phase, we generate a set $M$ of all possible candidate matching pairs, which leads to a large number of candidates i.e., $|M|=n *(n-1) / 2$. Then, we extract the attribute-value pairs using the feature extraction model and calculate the feature similarity vectors. In the final step we apply the previously built classification model to identify the matching pairs of products.

\subsection{Feature Extraction}

We pursue different approaches for extracting features from the structured and unstructured product descriptions. 


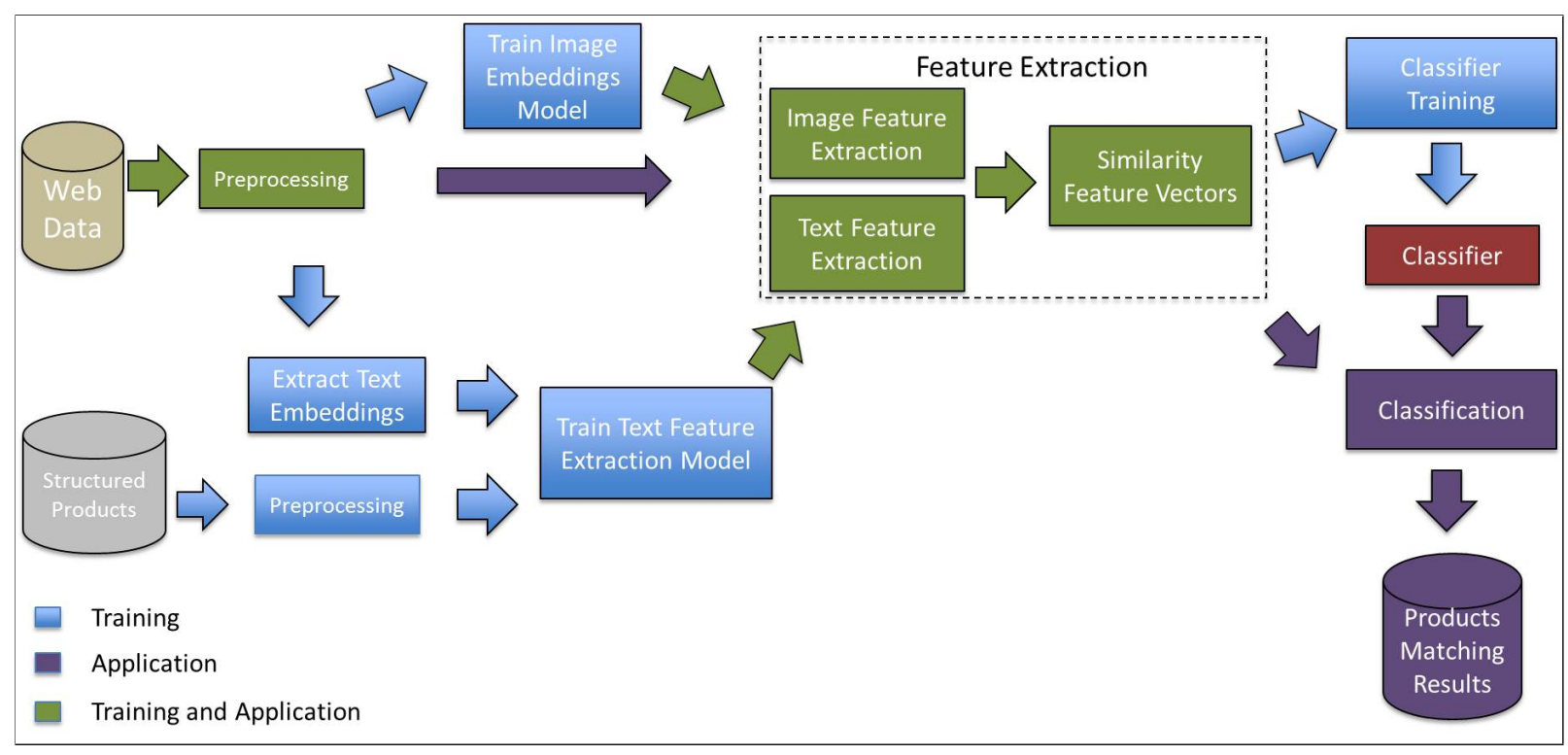

Fig. 1. System architecture overview

\subsubsection{Dictionary-Based Approach}

To implement the dictionary-based approach we were motivated by the approach described by Kannan et al [9]. We use the database $A$ of structured products to generate a dictionary of attributes and values. Let $F$ represent all the attributes present in the product database $A$. The dictionary represents an inverted in$\operatorname{dex} D$ from $A$ such that $D(v)$ returns the attribute name $f \in F$ associated with a string value $v$. Then, to extract features from a given product description $p \in P$, we generate all possible n-grams $(n \leq 4)$ from the text, and try to match them against the dictionary values. In case of multiple matches, we choose the longest ngram, and ties with multiple n-grams of the same maximal lengths are resolved by random selection.

\subsubsection{Conditional Random Fields}

A commonly used approach for tagging textual descriptions in NLP are conditional random field (CRF) models. A CRF is a conditional sequence model which defines a conditional probability distribution over label sequences given a particular observation sequence. In this work we use the Stanford CRF implementation ${ }^{6}$ in order to train product specific CRF models [4]. To train the CRF model we use comprehensive set of discrete features that comes from the standard distribution of the Stanford NER model: current word, previous word, next word, current word character n-gram $(n \leq 6)$, cur-

\footnotetext{
${ }^{6}$ http://nlp.stanford.edu/software/CRE-NER.shtml
}

rent POS tag, surrounding POS tag sequence, current word shape, surrounding word shape sequence, presence of word in left window (size $=4$ ) and presence of word in right window (size $=4$ ).

While CRF delivers rather good results, it requires a lot of labeled and diverse data. This becomes a challenge when new products are emerging on the market everyday, and merchants are changing the textual pattern of the product description. To address this challenge, we make use of the available unstructured data. Specifically, we use neural language modeling to extract word embeddings from the unstructured product description. The goal of such approaches is to estimate the likelihood of a specific sequence of words appearing in a corpus, explicitly modeling the assumption that closer words in the word sequence are statistically more dependent. One of the most popular and widely used is the word2vec neural language model [21,22]. Word2vec is a particularly computationally-efficient two-layer neural net model for learning word embeddings from raw text. There are two different algorithms, the Continuous Bag-of-Words model (CBOW) and the Skip-Gram model. The Skip-Gram model predicts contextual words given window of size $\mathrm{n}$, while CBOW predicts the current word, given the context in the window.

Projecting such latent representation of words into a lower dimensional feature space shows that semantically similar words appear closer to each other (see Section 5.3). Meaning that values of different prod- 


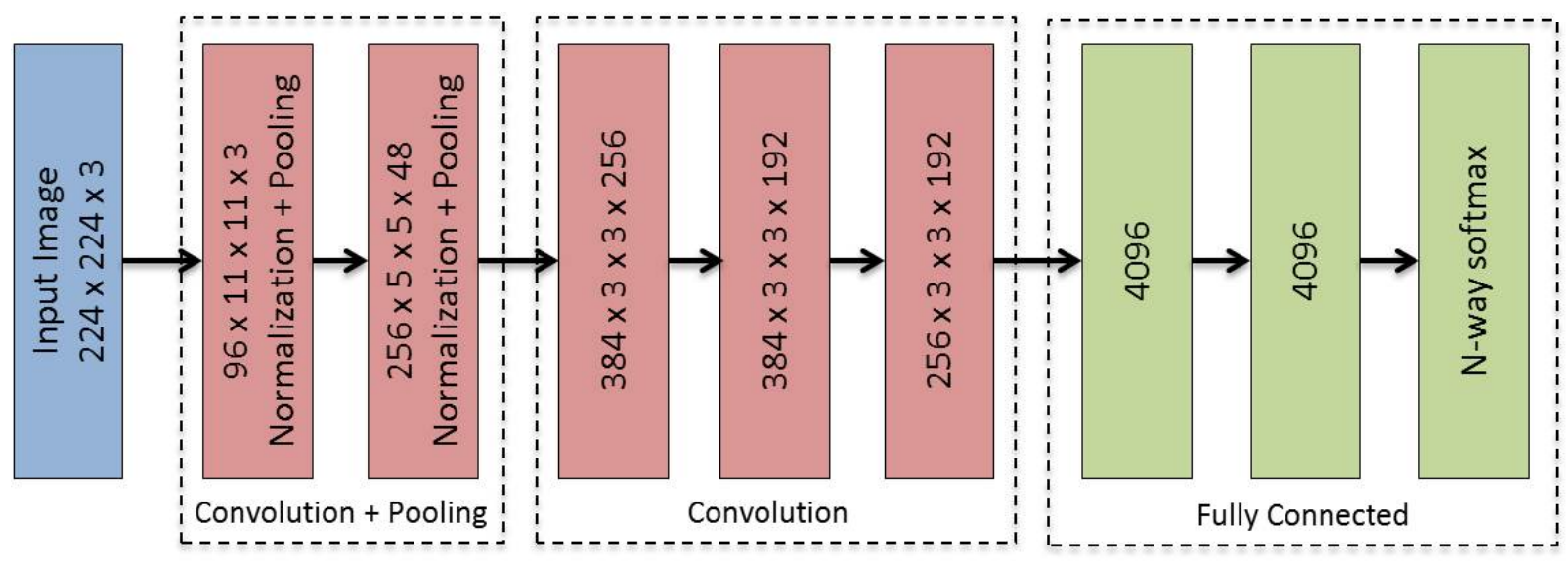

Fig. 2. Convolutional Neural Nets architecture

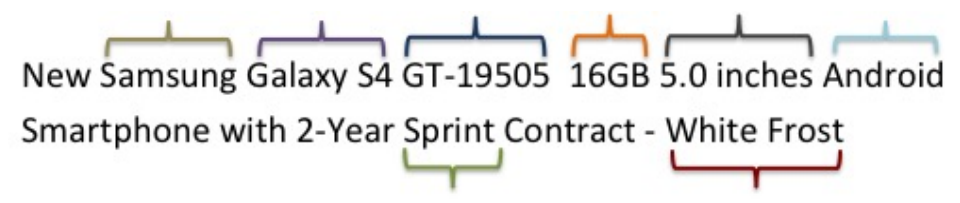

Fig. 3. Example of attribute extraction from a product title

Table 1

Attributes and values normalization

\begin{tabular}{|c|c|c|c|}
\hline Attribute Name & Attribute Value & Normalized Attribute Value & Attribute Data type \\
\hline \hline Brand & Samsung & samsung & string \\
\hline Phone type & Galaxy S4 & galaxy s4 & string \\
\hline Product code & GT-19505 & gt-19505 & string \\
\hline Memory & $16 \mathrm{~GB}$ & $1.6 \mathrm{e}+10(\mathrm{~B})$ & unit \\
\hline Size & 5.0 inches & $0.127(\mathrm{~m})$ & unit \\
\hline Operating system & Android & android & string \\
\hline Phone carrier & Sprint & sprint & string \\
\hline Color & White Frost & white frost & string \\
\hline Tagline & $\begin{array}{l}\text { New Smartphone } \\
\text { with 2-Year Contract }\end{array}$ & $\begin{array}{l}\text { new smartphone } \\
\text { with 2 year contract }\end{array}$ & long string \\
\hline
\end{tabular}

uct attributes are represented close to each other in the latent feature space. Therefore, we follow the approach presented in [29] to implement a CRF model that beside the previously described discrete features, includes the word embeddings as features for the CRF model. Training the CRF model using word embeddings makes the model more robust, meaning it is able to detect attributes that have not been seen during the training phase with higher precision.

\subsubsection{Image Feature Extraction}

Many e-shops use the same or similar image for identical products. Therefore, the image can be used as an indicator for identifying matching products. In this work, we use one of the most popular image pro- cessing techniques, deep Convolutional Neural Networks (CNNs) [15]. Usually, CNN models consist of several convolutional layers connected in a row, followed by fully connected layers. In each convolutional layer, several small filters are convolved on the input image. The weights of each filter are randomly initialized, thus, different filters get excited over different features in the image, e.g., some might get activated over a specific shape in the image, while other on a specific color. As this might produce a large number of features, each convolutional layer is usually connected to a pooling layer, which reduces the number of features by subsampling. The output of the convolutional layer is connected to a standard Feed-Forward Neural Net to solve a particular task. 
In this work, we adopt the same architecture proposed in [13]. The architecture of the model is shown in Figure 2. The network consist of five convolutional layers, followed by three fully connected layers. The number and size of the used filters in each convolutional layer is shown in the Figure. All neurons have a Rectified Linear Unit (ReLU) activation function to speed up the learning process. The output of the last fully-connected layer is fed to a $\mathrm{N}$-way softmax, where $\mathrm{N}$ is the number of labels in the dataset.

\subsubsection{Attribute Value Normalization}

Once all attribute-value pairs are extracted from the given dataset of offers, we continue with normalizing the values of the attributes. To do so, we use the same attribute normalzation pipeline presented in [26], i.e., attribute type detection, string normalization, number and number with unit of measurement normalization.

In Fig. 3 we give an example of feature extraction from a given product title. The extracted attributevalue pairs are shown in Table 1, as well as the normalized values, and the detected attribute data type.

\subsection{Calculating Similarity of Feature Vectors}

After the feature extraction is done, we can define an attribute space $F=\left\{f_{1}, f_{2}, \ldots, f_{n}\right\}$ that contains all of the extracted attributes, including the image vectors. To measure the similarity between two products we calculate similarity feature vector $F\left(p_{i}, p_{j}\right)$ for each candidate product pair. For two products $p_{1}$ and $p_{2}$, represented by the attribute vectors $F_{p 1}=\left\{f_{1} v_{1}, f_{2} v_{1}, \ldots, f_{n} v_{1}\right\}$ and $F_{p 2}=$ $\left\{f_{1} v_{2}, f_{2} v_{2}, \ldots, f_{n} v_{2}\right\}$, respectively, we calculate the similarity feature vector $F\left(p_{1}, p_{2}\right)$ by calculating the similarity value for each attribute $f$ in the attribute space $F$. Let $p_{1}$.val(f) and $p_{2} \cdot \operatorname{val}(\mathrm{f})$ represent the value of an attribute $f$ from $p_{1}$ and $p_{2}$, respectively. The similarity between $p_{1}$ and $p_{2}$ for the attribute $f$ is calculated based on the attribute data type as shown in Equation 1.

The Jaccard similarity is calculated on character ngrams $(n \leq 4)$, and the Cosine similarity is calculated on word tokens using TF-IDF weights.

$$
f\left(p_{1}, p 2\right)= \begin{cases}0, & \text { if } p_{1} \cdot \operatorname{val}(f)=0 \text { OR } p_{2} \cdot v a l(f)=0 \\ \text { JaccardSimilarity }\left(p_{1} \cdot \operatorname{val}(f), p_{2} \cdot \operatorname{val}(f)\right), & \text { if } f \text { is string attribute } \\ \text { CosineSimilarity }\left(p_{1} \cdot \operatorname{val}(f), p_{2} \cdot \operatorname{val}(f)\right), & \text { if } f \text { is long string attribute } \\ p_{1} \cdot \operatorname{val}(f)==p_{2} \cdot \operatorname{val}(f) \quad ? \quad 1: 0, & \text { if } f \text { is numeric or unit attribute } \\ \text { CosineSimilarity }\left(p_{1} \cdot \operatorname{val}(f), p_{2} \cdot \operatorname{val}(f)\right), & \text { if } f \text { is vector attribute (image embeddings) }\end{cases}
$$

\subsection{Classification Approaches}

Once the similarity feature vectors are calculated, we train four different classifiers: (i) Random Forest, (ii) Support Vector Machines (SVM), (iii) Naive Bayes and (iv) Logistic Regression.

\section{Product Categorization Approach}

The approach for product categorization consists of two steps: (i) feature extraction and (ii) classification. We use supervised and unsupervised approaches for feature extraction.

\subsection{Feature Extraction}

We use similar feature sets as we use for product matching.

\subsubsection{Supervised Text-based Feature Extraction}

For the task of product categorization we use a dictionary-based approach for feature extraction from product descriptions [26] $]^{7}$. The dictionary-based approach starts by building a dictionary of all attributevalue pairs used in the structured product dataset. To generate the feature vectors for each instance, after the features from the text are extracted, the value of each feature is tokenized, lowercased, and eliminated tokens shorter than 3 characters. The terms of each feature are concatenated with the feature name e.g. for the value blue for the feature color, the final value will be blue-color.

\footnotetext{
${ }^{7}$ We were not able to build a sufficiently good CRF model that is able to annotate text with high precision because of the many possible attributes across all categories. A separate CRF model for each category in the structured product dataset should be trained.
} 


\subsubsection{Unsupervised Text-based Feature Extraction}

Similarly to Section 3.3.2, we use neural language modeling to extract text embeddings from the unstructured product descriptions in order to overcome the challenge of the diversity of new products and their ever changing description. Since our product descriptions represent whole documents for the classification task we construct text embeddings given the whole document. The most prominent neural language model for text embedding on a document level is paragrph2vec [14], an extension to word2vec. As with word2vec, paragraph2vec relies on two algorithms: Distributed Memory (DM), which corresponds to CBOW, and Distributed Bag-of-Words (DBOW), which corresponds to the skip-gram model. Paragraph2vec is based on the same computationallyefficient two-layer neural network architecture. However, to be able to represent document embeddings paragrpah2vec maps each document to a unique paragraph vector. In DM this paragraph vector is averaged/summed with the word vectors, making the paragraph vector a memory of what is missing from the current context. Contrary to DM, DBOW ignores the context words in the input and instead forms a classification task given the paragraph vector and randomly selected words from a text window sample.

\subsubsection{Unsupervised Image-based Feature Extraction}

We use the same CNN model for extracting image embeddings as described in Section 3.3.1. In this case, we use the image vectors as such, i.e., we use the complete image vectors for the task of image classification.

\subsection{Classification}

For each of the feature extraction approaches in the end we use the feature vectors to build a classification model, i.e., Naive Bayes (NB), Support Vector Machines (SVM), Random Forest (RF) and k-Nearest Neighbors (KNN), where $\mathrm{k}=1$.

\section{Products Matching Evaluation}

\subsection{Datasets}

For the evaluation, we use Yahoo's Gemini Product Ads (GPA) for supervision ${ }^{8}$, and we use a subset of the WebDataCommons (WDC) extraction ${ }^{9}$.

\footnotetext{
${ }^{8}$ Note: we could use any database of structured products for the given task

${ }^{9}$ http: //webdatacommons.org/structureddata/index. html
}

Table 4

Datasets used in the evaluation

\begin{tabular}{|c|c|c|c|}
\hline Dataset & \#products & \#matching pairs & \#non-matching pairs \\
\hline Laptops & 209 & 146 & 25,521 \\
\hline Televisions & 344 & 236 & 58,760 \\
\hline Mobile Phones & 225 & 467 & 24,734 \\
\hline
\end{tabular}

\subsubsection{Product Ads - GPA dataset}

For our experiments, we are using a sample of three product categories from the Yahoo's Gemini Product Ads database. More precisely, we use a sample of 3,330 laptops, 3,476 TVs, and 3,372 mobile phones. There are 35 different attributes in the TVs and mobile phones categories, and 27 attributes in the laptops category. We use this dataset to build the Dictionary and the CRF feature extraction models.

\subsubsection{Unstructured Product Offers - WDC Microdata Dataset}

The latest extraction of WebDataCommons includes over 5 billion entities marked up by one of the three main HTML markup languages (i.e., Microdata, Microformats and $\mathrm{RDFa}$ ) and has been retrieved from the CommonCrawl 2014 corpus $^{10}$. From this dataset we focus on product entities annotated with Microdata using the schema.org vocabulary. To do so, we use a sub-set of entities annotated with $\mathrm{s}:$ Product. The dataset contains 288,082,823 entities in total, or 2,829,523,589 RDF quads. 89,608 PLDs (10.9\%) annotate at least one entity as $\mathrm{s}:$ Product and 62,849 PLDs $(7.6 \%)$ annotate at least one entity as $\mathrm{s}$ : Offer. In our approach, we make use of the properties $s:$ name and $s$ :description for extracting attribute-value pairs, and the $\mathrm{s}:$ Product/image image embeddings.

To evaluate the approach, we built a gold standard from the WDC dataset on three categories in the Electronics domain, i.e., TVs, mobile phones and laptops. We set some constraints on the entities we select: (i) the products must contain an $s:$ name and an $s$ : description property in English language, (ii) the $s$ : name must contain between 3 and 50 words, (iii) the $s$ :description must contain between 10 and 200 words, (iv) ignore entities from community advertisement websites (e.g., gumtree.com), (v) the product can be uniquely identified based on the title and description i.e., it contains enough information to pinpoint the exact product.

\footnotetext{
${ }^{10}$ http://blog.commoncrawl.org/2015/01/ december-2014-crawl-archive-available/
} 
Table 2

CRF evaluation on GPA data

\begin{tabular}{|c|c|c|c||c|c|c||c|c|c||c|}
\hline & & & \multicolumn{1}{|c||}{} & \multicolumn{3}{c||}{ CRF } & \multicolumn{3}{c||}{ CRFemb } & \\
\hline Dataset & \#training & \#test & \#atts. & P & R & F1 & P & R & F1 & $\Delta$ F1 \\
\hline Laptops & 2,330 & 1,000 & 27 & 94.81 & 93.35 & $\mathbf{9 4 . 0 8}$ & 93.67 & 93.2 & 93.43 & -0.65 \\
\hline Televisions & 2,436 & 1,040 & 35 & 96.2 & 94.31 & 95.25 & 96.41 & 94.85 & $\mathbf{9 5 . 6 2}$ & 0.37 \\
\hline Mobile Phones & 2,220 & 1,010 & 35 & 97.62 & 96.13 & $\mathbf{9 6 . 8 7}$ & 96.72 & 95.84 & 96.27 & -0.6 \\
\hline
\end{tabular}

Table 3

CRF evaluation on WDC data

\begin{tabular}{|c|c||c|c|c||c|c|c||c|}
\hline & \multicolumn{1}{|c||}{} & \multicolumn{3}{c||}{ CRF } & \multicolumn{3}{c|}{ CRFemb } & \\
\hline Dataset & \#test & $\mathrm{P}$ & $\mathrm{R}$ & $\mathrm{F} 1$ & $\mathrm{P}$ & $\mathrm{R}$ & $\mathrm{F} 1$ & $\Delta \mathrm{F} 1$ \\
\hline Laptops & 50 & 57.71 & 53.95 & 55.76 & 71.91 & 64.19 & $\mathbf{6 7 . 8 3}$ & 12.06 \\
\hline Televisions & 50 & 84.62 & 56.57 & 67.81 & 88.24 & 77.14 & $\mathbf{8 2 . 3 2}$ & 14.51 \\
\hline Mobile Phones & 50 & 72.83 & 60.12 & 65.87 & 85.03 & 64.25 & $\mathbf{7 3 . 2}$ & 7.33 \\
\hline
\end{tabular}

The gold standard is generated by manually identifying matching products in the whole dataset. Two entities are labeled as matching products if both entities contain enough information to be uniquely identified, and both entities point to the same product. It is important to note that the entities do not necessarily contain the same set of product features. The number of entities, the number of matching and non-matching pairs for each of the datasets is shown in Table 4.

To build the text embeddings models we select two subsets of the unstructured data: (i) subset containing only the $s$ : name, (ii) subset containing the $s$ : name and $s$ :description. Additionally, we preprocess the WDC dataset even further, i.e., we tokenize the input such that complex product IDs (ex. G70-3580Q5002SGE) are considered as one word, and we apply a WordNet ${ }^{11}$ lemmatizer.

\subsection{CRF Evaluation}

Here, we compare the performances of the CRF model built only on discrete features (just CRF in the following) with the CRF model built on discrete features and continuous features from word embeddings (CRFemb in the following). To train the CRFemb model we build both CBOW and Skip-Gram neural models for word embeddings. We train the models on the complete WDC and GPA datasets. We experimented with different parameters for the models, to finally select the following parameters' values: window size $=5$; number of iterations $=10$; negative sampling for optimization; negative samples $=10$; with average input vector for CBOW; vectors size $=200$. We

\footnotetext{
${ }^{11}$ https://wordnet.princeton.edu/
}

used the gensim implementation ${ }^{12}$ for model training. All models, as well as an extended overview of the parameters are available for download online ${ }^{13}$.

We evaluate both CRF models on the database of structured product ads. For each of the three product categories we select $70 \%$ of the instances as a training set and the rest as a test set. The results for each category, as well as the number of instances used for training and testing, and the number of attributes are shown in Table 2.

The results show that there is no significant difference in the performance of the two models. However, we believe that the models might be overfitted on the structured product ads. The reason for this assumption is that the structured product ads are rather clean, and ads coming from the same retailer follow similar patterns. Consequently, we are not able to observe significant difference in performance between the two models. Therefore, we manually labeled all the attributevalue pairs in 50 products of each category on WDC products. The evaluation results of both models on the new data are shown in Table 3 . The results show significant difference in performance between the two models. The reason for such difference in performance is that the CRFemb model is more robust because of the word embedding features, allowing it to identify attribute-value pairs in "dirty" data from the Web.

\subsection{Semantics of Vector Representations}

To analyze the semantics of the word vector representations, and get deeper insigts of their relevance for training the CRF model, we employ Principal Compo-

\footnotetext{
${ }^{12}$ https://radimrehurek.com/gensim/

${ }^{13} \mathrm{http}: / /$ data.dws.informatik.uni-mannheim.de/gpawdc/DL
} 


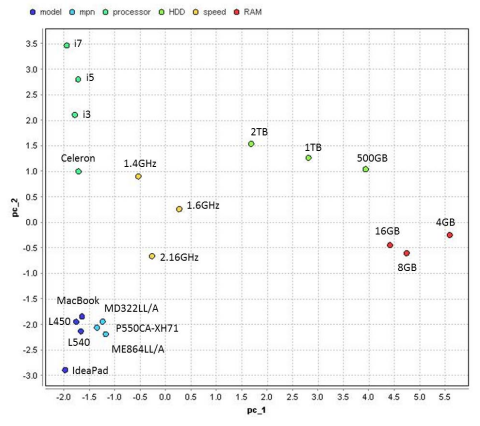

a) Laptop attributes

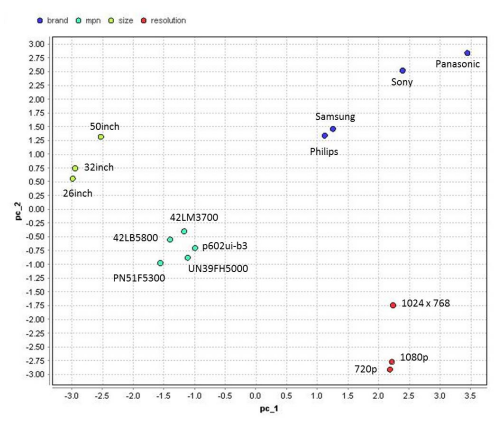

b) TV attributes

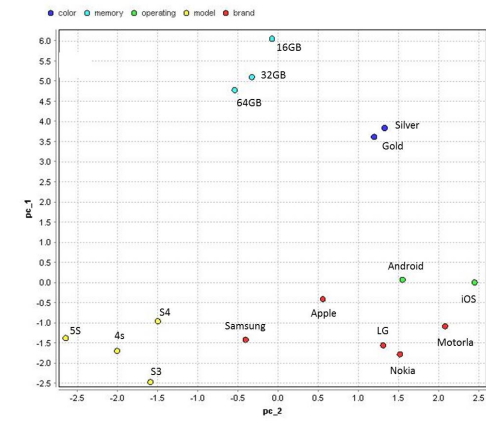

c) Mobile attributes

Fig. 4. Two-dimensional PCA projection of the 200-dimensional Skip-Gram vectors for different product attributes, extracted from the product titles.

nent Analysis (PCA) to project the"high"-dimensional attribute vectors in a two dimensional feature space. We select several attribute-value pairs for each category and plotted them on a 2D scatter plot, as shown in Figure 4. The figure illustrates the ability of the model to automatically organize values of different attributes, i.e., values of the same attributes are usually positioned close to each other. Also, the model was able to automatically order semantically close attributes (for instance processor is closest to speed shown in Figure 4a).

\subsection{Experiment Setup}

To evaluate the effectiveness of the product matching approach we use the standard performance measures, i.e., Precision (P), Recall (R) and F-score (F1). The results are calculated using stratified 10-fold cross validation. For conducting the experiments, we used the RapidMiner machine learning platform and the RapidMiner development library. ${ }^{14}$ We compare the results of the product matching approach when using the Dictionary approach, the CRF and the CRFemb model for feature extraction. Furthermore, we add image embeddings as a weaker signal for the given task. To do so, we train the CNN model described in Section 3.3.1, using a dataset of 8,362 products images, labeled with 303 different labels from the third level of the GS1 product catalogue (see Section 7.1). We use this CNN model as an image feature extraction model. To do so, for each image we use the output of the second fully connected layer, which results in a feature vector of length 4,096. Such image vectors can be used in any classification algorithm. For each pair of prod-

\footnotetext{
${ }^{14}$ http: //www.rapidminer.com/
}

ucts we calculate the cosine similarity on the image vectors, which is included in the feature similarity vector for the given pair of products.

We compare our approach to three baselines. As a first baseline, we match the products based on a Bagof-Words TF-IDF cosine similarity, reporting the best score on different levels of matching thresholds, i.e., we iterate the matching threshold starting from 0.0 to 1.0 (with step 0.01) and we assume that all pairs with similarity above the threshold are matching pairs. We calculated the similarity based on different combination of title and description, but the best results were delivered when using only the product title.

As a second baseline we use the document vectors generated as explained in section 4.1.2. Moreover, we build both DM and DBOW models for each of the datasets. We experiment with different vectors size, i.e., 50, 200, 500 and 1000 . We calculate the cosine similarity between each pair of vectors, and we report the best score on different levels of matching thresholds.

As a third baseline, we use the Silk Link Discovery Framework [8], an open-source tool for discovering links between data items within different data sources. The tool uses genetic algorithm to learn linkage rules based on the extracted attributes. For this experiment, we first extract the features from the product title and description using our CRF model, and then represent the gold standard in RDF format. The evaluation is performed using 10-fold cross validation.

\subsection{Results}

The results for the baselines are shown in Table 5 . For all three approaches the best results are achieved when using only the title of the products. The best 
Table 5

Products matching baseline results using cosine similarity on TF-IDF, paragraph2vec and Silk

\begin{tabular}{|c|c|c|c|c|c|c|c|c|c|}
\hline & \multicolumn{3}{|c|}{ Laptops } & \multicolumn{3}{c|}{ Television } & \multicolumn{3}{c|}{ Mobile Phones } \\
\hline Approach & $\mathrm{P}$ & $\mathrm{R}$ & $\mathrm{F} 1$ & $\mathrm{P}$ & $\mathrm{R}$ & $\mathrm{F} 1$ & $\mathrm{P}$ & $\mathrm{R}$ & $\mathrm{F} 1$ \\
\hline Cosine similarity TF-IDF (title) & 29.6 & 39.7 & 33.9 & 29.9 & 21.9 & 25.3 & 37.5 & 38.3 & 37.9 \\
\hline Doc2Vec title (DBOW 50) & 35.8 & 32.9 & 34.3 & 31.2 & 23.4 & 26.7 & 73.0 & 32.8 & 45.3 \\
\hline Silk & 28.4 & 80.8 & $\mathbf{4 2 . 0}$ & 50.1 & 91.1 & $\mathbf{6 4 . 6}$ & 40.6 & 84 & $\mathbf{5 4 . 7}$ \\
\hline
\end{tabular}

Table 6

Product Matching Performance

\begin{tabular}{|c|c|c|c|c|c|c|c|c|c|c|c|c|}
\hline \multirow[t]{2}{*}{ Model } & \multicolumn{3}{|c|}{ Dictionary } & \multicolumn{3}{|c|}{$\mathrm{CRF}$} & \multicolumn{3}{|c|}{ CRFemb } & \multicolumn{3}{|c|}{ CRFemb + Image emb. } \\
\hline & $\mathrm{P}$ & $\mathrm{R}$ & F1 & $P$ & $\mathrm{R}$ & F1 & $P$ & $\mathrm{R}$ & F1 & $\mathrm{P}$ & $\mathrm{R}$ & F1 \\
\hline \multicolumn{13}{|c|}{ Laptops } \\
\hline Random Forest & 74.1 & 49.81 & 59.56 & 81.52 & 51.29 & 62.96 & 72.85 & 71.33 & 72.08 & 81.82 & 66.47 & 73.35 \\
\hline SVM & 72.49 & 50.29 & 59.56 & 69.42 & 56.48 & 62.28 & 84.99 & 54.76 & 66.61 & 79.50 & 60.62 & 68.79 \\
\hline Naive Bayes & 11.61 & 55.95 & 19.23 & 6.20 & 75.81 & 11.46 & 7.11 & 83.57 & 12.87 & 6.31 & 84.29 & 11.56 \\
\hline Logistic Regression & 56.06 & 47.52 & 51.44 & 65.05 & 51.02 & 57.18 & 51.00 & 79.20 & 62.05 & 53.00 & 78.30 & 63.21 \\
\hline \multicolumn{13}{|c|}{ Televisions } \\
\hline Random Forest & 80.53 & 74.13 & 77.20 & 92.10 & 73.90 & 82.00 & 83.65 & 82.74 & 83.19 & 93.06 & 78.16 & 84.96 \\
\hline SVM & 62.20 & 62.97 & 62.58 & 84.90 & 63.90 & 72.90 & 84.90 & 71.68 & 77.73 & 85.71 & 73.03 & 78.86 \\
\hline Naive Bayes & 7.43 & 94.12 & 13.74 & 7.26 & 89.37 & 13.36 & 5.45 & 95.78 & 10.28 & 6.31 & 93.66 & 11.72 \\
\hline Logistic Regression & 23.22 & 81.13 & 36.14 & 51.92 & 76.93 & 61.94 & 52.74 & 77.62 & 62.80 & 51.59 & 79.82 & 62.67 \\
\hline \multicolumn{13}{|c|}{ Mobile Phones } \\
\hline Random Forest & 38.6 & 65.92 & 48.68 & 88.49 & 75.60 & 81.53 & 89.42 & 77.01 & 82.75 & 90.56 & 77.06 & 83.27 \\
\hline SVM & 41.60 & 16.26 & 23.38 & 43.95 & 45.65 & 44.78 & 44.81 & 45.58 & 45.19 & 46.46 & 45.67 & 46.06 \\
\hline Naive Bayes & 10.20 & 35.36 & 15.83 & 15.31 & 63.06 & 24.63 & 15.46 & 73.23 & 25.53 & 15.37 & 76.74 & 25.61 \\
\hline Logistic Regression & 27.85 & 32.50 & 29.99 & 41.34 & 48.93 & 44.82 & 43.36 & 47.29 & 45.23 & 44.66 & 47.4 & 45.98 \\
\hline
\end{tabular}

results for the paragraph2vec approach are achieved when using the DBOW method with 50 latent features. We can see that the documents embeddings outperform the standard BOW TF-IDF, and the Silk framework outperforms both. However, we might conclude that both baseline approaches deliver rather poor results.

Next, we show the results for products matching using the CRFemb for attribute extraction, compared to the standard CRF and the Dictionary model. The results are given in Table 6. We can note that all three approaches significantly outperform the baseline approaches. The Random Forest classifier delivers the best result for all three categories using the CRFemb feature extraction approach. We can observe that the other classifiers achieve high recall, i.e., they are able to detect the matching pairs in the dataset, but they also misclassify a lot of non-matching pairs, leading to a low precision. It is noteworthy that the results for the laptops datasets are significantly better when using the CRFemb approach, compared to the CRF and the Dictionary approach. We already showed in [26] that the matching task for laptops is more challenging, because it needs more overlapping features to conclude that two products are matching ${ }^{15}$. The improvement of the results confirms that the CRFemb is more robust than the CRF and the Dictionary approach, and it is able to extract attributes with higher precision. Furthermore, we can note that the results using the dictionarybased approach are significantly worse than the CRF approach. The reason is that the GPA dataset contains a lot of phones that were advertised in the year 2015, while the WDC dataset contains phones that were popular in 2014. Therefore, the dictionary-based approach fails to extract many attribute-value pairs.

From the last column of the table, we can conclude that using the image embeddings slightly improves the results. Furthermore, the feature relevance analysis using information gain shows a rather high relevance of the image similarity feature for the given task, and it is comparable with the relevance of the brand attribute. The image cannot be used as a strong signal for the task of product matching because a lot of similar products of the same brand have the same or similar image. For example, "iPhone 6s 16GB gold" and"iPhone 6s 64GB gold" have the same image across many eshops. However, using such image features it is pos-

\footnotetext{
${ }^{15}$ For example, two laptops might share the same brand, same CPU, and same HDD, but if the memory differs, then the laptops are not the same.
} 


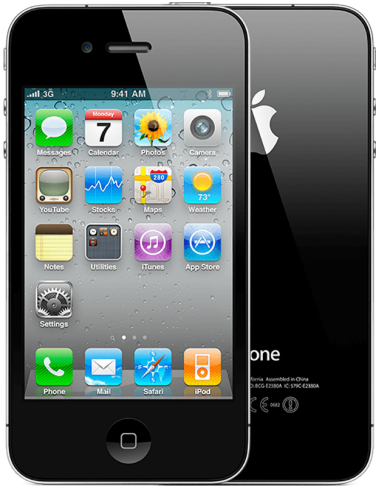

a) iPhone $416 G B$
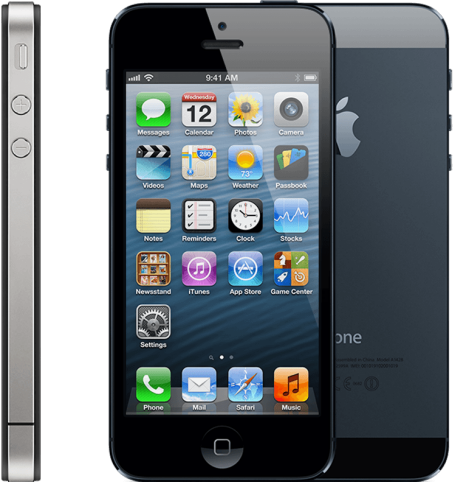

b) iPhone $516 G B$
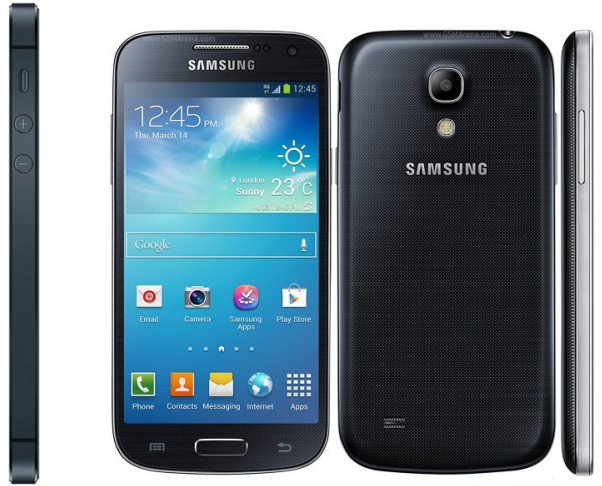

c) Samsung Galaxy S4 Mini

Fig. 5. Images for Products

sible to distinguish products of different brands. For example, in Fig 5a is given an iPhone $416 G B$, in Fig $5 \mathrm{~b}$ is given an iPhone $516 \mathrm{~GB}$ and in Fig $5 \mathrm{c}$ is given an Samsung Galaxy S4 Mini. The cosine similarity of the image features between the first two is 0.76 , while the cosine similarity between the first and third mobile phone is 0.35 .

\section{Use Case: Enriching Product Ads with Semantic Structured Data}

Product ads are a popular form of search advertizing $^{16}$ that are increasingly used as a replacement for text-based search ads, and are currently offered as an option by Bing, Google and Yahoo under different trade names. Unlike traditional search ads that carry only a title, link and a description, product ads are more structured. They often include further details such as the product identifier, brand, model for electronics, or gender for clothing. These details are provided as part of data feeds that merchants transmit to the search engine, and they allow search engine providers to perform better keyword-based ad retrieval, and to offer additional options such as faceted search over a set of product results. The level of completeness of the product specification, however, depends on the completeness of the advertisers' own data, their level of technical sophistication in creating data feeds and/or willingness to provide additional information to the search engine provider beyond the minimally required set of attributes. As a result of this,

\footnotetext{
${ }^{16}$ Search advertising is a method of placing online advertisements on web pages that show results from search engine queries.
}

product ads are often very incomplete when it comes to the details of the product on offer.

In this section, we apply the previously built models on the whole WDC and GPA products datasets, in order to identify product matches for the products in the GPA dataset, and extract additional attributes from the WDC products. First, we try to identify duplicate products within the WDC dataset for top $10 \mathrm{TV}$ brands. Then, we try to identify matching products in the WDC dataset for the product ads in the GPA dataset in the TV category. To do so, we use the best performing models evaluated in the previous section, i.e., a Random Forest model built on CRF features, and a Random Forest model built on CRFemb features. We do not include the Dictionary approach, because the initial experiments yield rather poor results.

\subsection{Integrating Unstructured Product Descriptions}

In the first experiment, we apply the models to identify matching products for the top $10 \mathrm{TV}$ brands in the WDC dataset. To do so, we selected a sub-set of products from the WDC dataset that contain one of the TV brands in the s:name or s:description of the products. Furthermore, we apply the same constraints described in Section 5, which reduces the number of products. We use the brand name as a blocking approach, i.e., we generate candidate matching pairs only for products that share the same brand. We use the CRF and CRFemb feature extraction approaches to extract the features separately, and we tune the Random Forest model in a way that we increase the precision, on the cost of lower recall, i.e., a candidate products pair is considered to be positive matching pair if the classification confidence of the model is above 0.8 . 
Table 7

Discovered matching products in the WDC dataset

\begin{tabular}{|l|r||r|r||r|r|}
\hline & \multicolumn{1}{|c||}{} & \multicolumn{2}{|c||}{ CRF } & \multicolumn{2}{c|}{ CRFemb } \\
\hline Brand & \#WDC products & \#Matches & Precision & \#Matches & Precision \\
\hline sony & 3,673 & 926 & 96.00 & 973 & 95.58 \\
\hline $\lg$ & 14,764 & 734 & 94.00 & 758 & 94.19 \\
\hline samsung & 4,864 & 567 & 88.18 & 587 & 89.43 \\
\hline rca & 3,961 & 385 & 93.55 & 401 & 93.51 \\
\hline vizio & 563 & 296 & 94.59 & 271 & 97.78 \\
\hline panasonic & 1,696 & 160 & 93.75 & 173 & 94.21 \\
\hline philips & 1,466 & 44 & 95.45 & 49 & 95.91 \\
\hline magnavox & 141 & 29 & 100.00 & 39 & 97.43 \\
\hline nec & 23,845 & 18 & 100.00 & 26 & 100 \\
\hline proscan & 30 & 7 & 100.00 & 9 & 100 \\
\hline
\end{tabular}

Table 8

Discovered matching products in the WDC dataset for product ads in the GPA dataset

\begin{tabular}{|l|r|r||r|r||r|r|}
\hline & & \multicolumn{2}{|c||}{} & \multicolumn{2}{|c|}{ CRF } & \multicolumn{2}{c|}{ CRFemb } \\
\hline Brand & \#GPA products & \#WDC products & \#Matches & Precision & \#Matches & Precision \\
\hline samsung & 560 & 4,864 & 202 & 80.85 & 217 & 82.02 \\
\hline vizio & 253 & 563 & 123 & 91.80 & 161 & 92.54 \\
\hline lg & 288 & 14,764 & 102 & 89.24 & 124 & 91.93 \\
\hline rca & 10 & 3,961 & 67 & 79.10 & 79 & 81.01 \\
\hline sony & 102 & 3,673 & 40 & 97.50 & 70 & 94.28 \\
\hline proscan & 18 & 30 & 28 & 100.00 & 29 & 100.00 \\
\hline nec & 22 & 23,845 & 21 & 85.70 & 33 & 87.87 \\
\hline magnavox & 28 & 141 & 12 & 100.00 & 27 & 100.00 \\
\hline panasonic & 41 & 1,696 & 6 & 100.00 & 10 & 100.00 \\
\hline philips & 11 & 1,466 & 2 & 100.00 & 10 & 100.00 \\
\hline
\end{tabular}

We report the number of discovered matches for each of the TV brands in Table 7. The second row of the table shows the number of candidate product descriptions after we apply the selection constraints on each brand. We manually evaluated the correctness of the matches and report the precision for both the $\mathrm{CRF}$ and the CRFemb approach. The results show that we are able to find a large number of matching products with high precision. The number of discovered matches when using the CRFemb approach is slightly higher compared to the CRF approach, while the precision remains in the same range. By relaxing the selection constraints of product candidates the number of discovered matches would increase, but it might also reduce the precision.

\subsection{Enriching Product Ads}

In this experiment we try to identify matching products in the WDC dataset for the product ads in the GPA dataset. Similarly as before, we select WDC products based on the brand name and we apply the same filtering to reduce the sub-set of products for matching. To extract the features for the WDC products we use the CRF and the CRFemb feature extraction models, and for the GPA products we use the already existing features provided by the merchants. To identify the matches we apply the respective Random Forest models for the CRF and the CRFemb approach. The results are shown in Table 8. The second row reports the number of products of the given brand in the GPA dataset, and the third row in the WDC dataset.

The results show that we are able to identify a small number of matching products with high precision. Again, the number of discovered matching products is slightly higher when using the CRFemb approach compared to the CRF approach, i.e., using the CRF approach we are able to find at least one match for 269 products in the GPA dataset, while using CRFemb for 310 products, and using the CRF approach there are 534 correct matches in total, while with CRFemb there are 676 correct matches. However, we have to note that we are not able to identify any matches for the products in the GPA dataset that are released after 2014, because they do not appear in the WDC dataset. Furthermore, we analyzed the number of new attributes we can discover for the GPA products from the matching WDC products. The distribution of matches, newly discovered attribute-value pairs, offers, ratings and reviews per GPA instance using the CRF approach is shown in 


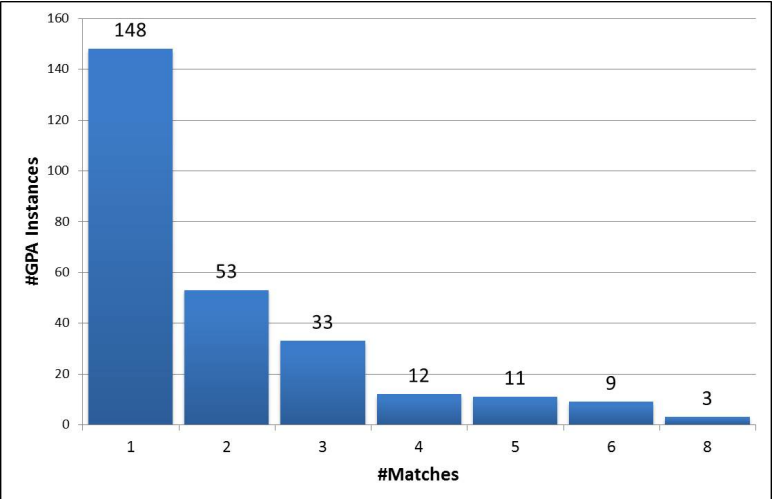

a) Matches distribution

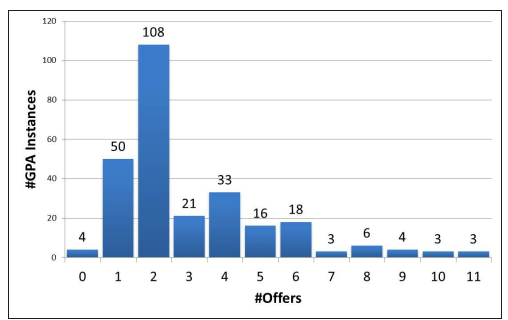

c) Offers distribution

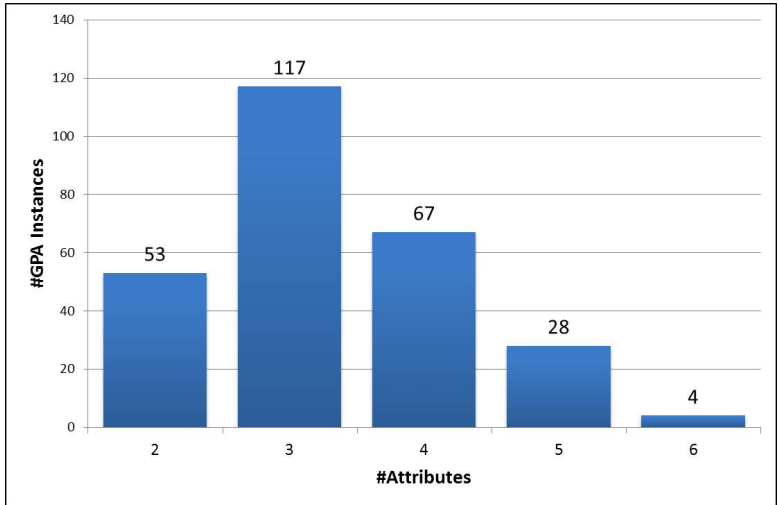

b) Attributes distribution

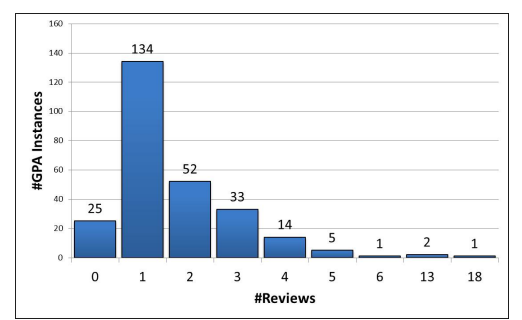

e) Reviews distribution

Fig. 6. Distribution of newly discovered matches and attributes per product ad using the CRF approach

Fig. 6. The results show that for each of the product ads that we found a matching product description, at least 1 new attribute-value pair was discovered. And for some product ads even 8 new attribute-value pairs were discovered. The same distributions when using the CRFemb approach are shown in Fig. 7.

\section{Product Categorization Evaluation}

In a second series of experiments, we evaluate the quality of our approach for product categorization.

\subsection{Dataset}

For our experiments we use the GS1 Product Cata$\log (\mathrm{GPC})^{17}$ as a target product categorization hierarchy. The hierarchy is structured in six different levels, but in our experiments we try to categorize the products in the first three levels of the taxonomy: Segment, Family and Class. The first level contains 38 different categories, the second level 113 categories (77 used) and the third level 783 categories (303 used).

\footnotetext{
${ }^{17}$ http: //www.gs1.org/gpc
}

To evaluate the proposed approach we use the Microdata products gold standard developed in [20]. We removed non-English instances from the dataset, resulting in 8,362 products. In our evaluation we use the s:name and the s:description properties for generating the text embeddings, and the s:Product/image for generating the image embeddings.

\subsection{Experiment Setup}

The evaluation is performed using 10-fold cross validation. We measure accuracy (Acc), Precision (P), Recall $(\mathrm{R})$ and F-score (F1). Here, we evaluate both supervised and unsupervised feature extraction for product categorization. Moreover, we compare the dictionary approach (Dict.) to the unsupervised paragraph2vec feature extraction (Par2vec), and the unsupervised image feature extraction model (ImgEmb). As for the product matching task (see Section 5.4), we build both DM and DBOW models with vector size of 50, 200, 500 and 1000.

For the image feature extraction we use a CNN model trained on the labels from the third level of the GS 1 catalog (ImgEmb). Furthermore, we compare our CNN model to an existing CNN model. In particular, 


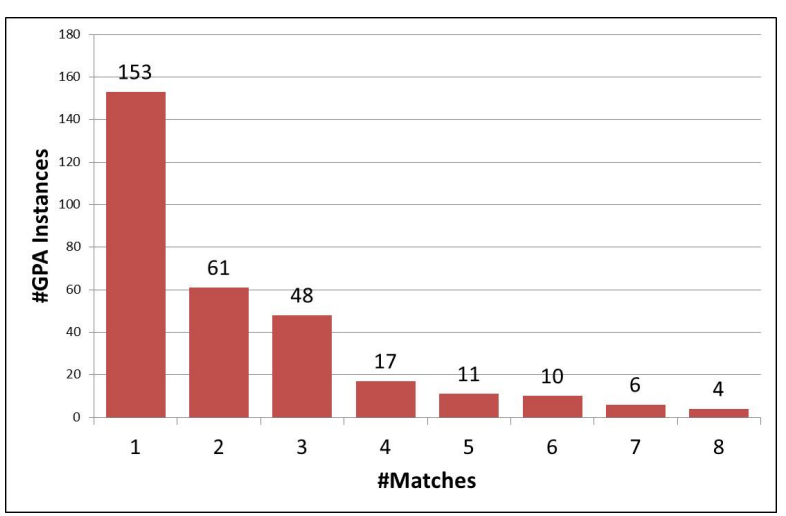

a) Matches distribution

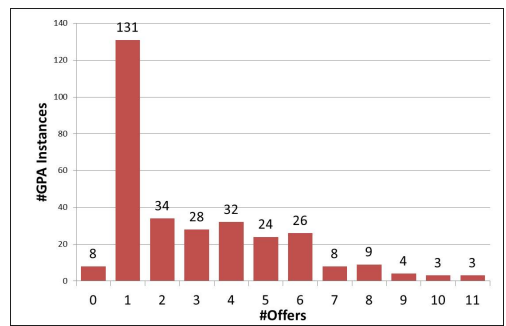

c) Offers distribution

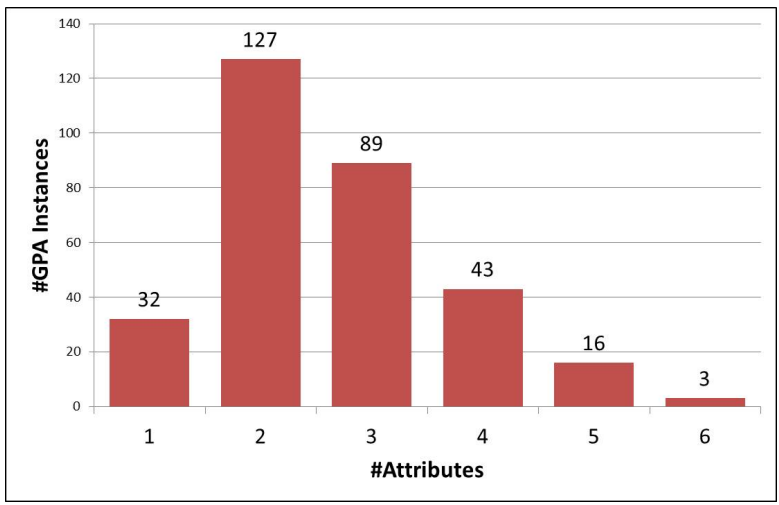

b) Attributes distribution

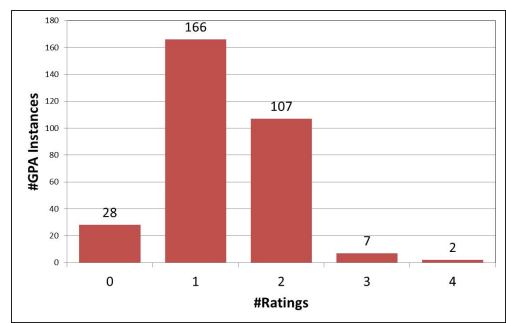

d) Ratings Distribution

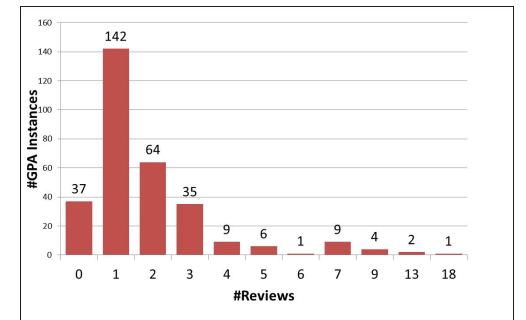

e) Reviews distribution

Fig. 7. Distribution of newly discovered matches and attributes per product ad using the CRFemb approach

we used a Caffe reference model ${ }^{18}$, which has been pre-trained on 1.2 million ImageNet (ILSVRC2012 challenge) images ${ }^{19}$. We use the output of the second fully connected layer, which results in a feature vector of length 4,096. Finally, we do a combination of both the unsupervised text feature extraction model and the image embedding model, by concatenating the output vectors of both models (Par2Vec + ImageNet).

We compare all of the approaches with a baseline approach based on a Bag-of-Words TF-IDF cosine similarity.

\subsection{Results}

The results for each of the three levels are shown in Table 9. All the experiments that did not finish within ten days, or that have run out of memory are marked with "I". We show only the best performing results of all the paragraph2vec models, where on each of the three levels the best results were achieved using the DBOW model with 500 dimensions, trained on both

\footnotetext{
${ }^{18}$ bvlc_reference_caffenet from caffe.berkeleyvision.org

${ }^{19}$ http://image-net.org/challenges/LSVRC/2012/
}

the title and description of the products. The complete results can be found online ${ }^{20}$.

We can observe that the supervised dictionary-based approach outperforms the rest on the first level. However, on the second and the third level the combination of the document and image embedding approach outperforms the others. Also, the documents embedding approach alone outperforms the baseline on the second and third level, and gives comparable results on the first level. It is interesting to note that the image embeddings approach alone performs rather well on all three levels, where the model trained only on product images performs slightly worse than the model built on the ImageNet dataset. The reason is that the number of labeled products images we used is significantly lower that the dataset used for training the ImageNet model. Also, we have to note that we did not apply any preprocessing or filtering on the images ${ }^{21}$.

\footnotetext{
${ }^{20}$ http://data.dws.informatik.uni-mannheim.de/ gpawdc/ISWC16

${ }^{21}$ Many images do not directly correspond to the product, or are of a bad quality.
} 
Table 9

Product Categorization results. The best results are marked in bold.

\begin{tabular}{|c|c|c|c|c|c|c|c|c|c|c|c|c|c|}
\hline & & \multicolumn{4}{|c|}{ GS1 Level 1} & \multicolumn{4}{|c|}{ GS1 Level 2} & \multicolumn{4}{|c|}{ GS1 Level 3} \\
\hline Features & Model & ACC & $P$ & $\mathrm{R}$ & F1 & ACC & $\mathrm{P}$ & $\mathrm{R}$ & F1 & ACC & $\mathrm{P}$ & $\mathrm{R}$ & F1 \\
\hline \multirow{4}{*}{$\begin{array}{c}\text { BoW } \\
\text { TF-IDF }\end{array}$} & NB & 80.87 & 61.26 & 51.64 & 56.04 & 78.81 & 42.21 & 37.78 & 39.87 & 69.87 & 20.47 & 22.70 & 21.52 \\
\hline & K-NN & 77.79 & 58.35 & 48.42 & 52.92 & 78.29 & 40.15 & 38.42 & 39.27 & 65.99 & 20.67 & 19.18 & 19.89 \\
\hline & SVM & 86.06 & 71.50 & 55.62 & 62.57 & 1 & 1 & 1 & 1 & 1 & 1 & 1 & 1 \\
\hline & $\mathrm{RF}$ & 73.58 & 55.52 & 36.74 & 44.22 & 73.00 & 39.43 & 27.96 & 32.72 & 65.35 & 20.12 & 16.87 & 18.35 \\
\hline \multirow{4}{*}{ Dict. } & NB & 81.98 & 66.43 & 51.68 & 58.13 & 79.87 & 46.31 & 40.08 & 42.97 & 70.90 & 25.45 & 24.36 & 24.89 \\
\hline & K-NN & 76.32 & 54.68 & 46.28 & 50.13 & 76.44 & 40.15 & 39.65 & 39.90 & 65.66 & 21.93 & 20.14 & 20.99 \\
\hline & SVM & 88.34 & 74.11 & 64.78 & 69.13 & 1 & 1 & 1 & 1 & 1 & 1 & 1 & 1 \\
\hline & RF & 79.07 & 66.73 & 49.09 & 56.57 & 73.74 & 41.75 & 31.93 & 36.19 & 65.82 & 21.87 & 18.61 & 20.10 \\
\hline \multirow{4}{*}{ Par2Vec } & NB & 67.01 & 46.39 & 44.90 & 45.63 & 65.59 & 34.73 & 33.57 & 34.14 & 65.82 & 20.52 & 21.40 & 20.95 \\
\hline & K-NN & 70.11 & 48.13 & 35.82 & 41.07 & 79.44 & 42.96 & 42.00 & 42.47 & 76.64 & 26.35 & 27.06 & 26.70 \\
\hline & SVM & 80.24 & 57.89 & 52.92 & 55.29 & 1 & 1 & 1 & 1 & 1 & 1 & 1 & 1 \\
\hline & $\mathrm{RF}$ & 58.17 & 41.40 & 16.50 & 23.60 & 68.82 & 31.80 & 25.69 & 28.42 & 62.63 & 16.15 & 14.80 & 15.45 \\
\hline \multirow{4}{*}{ ImgEmb } & NB & 56.85 & 34.04 & 23.69 & 27.94 & 55.39 & 21.92 & 13.97 & 17.06 & 51.26 & 14.72 & 8.31 & 10.62 \\
\hline & K-NN & 66.26 & 39.38 & 30.78 & 34.55 & 66.34 & 30.77 & 21.28 & 25.16 & 59.76 & 21.59 & 16.98 & 19.01 \\
\hline & SVM & 70.29 & 48.65 & 32.20 & 38.75 & 1 & 1 & 1 & 1 & 1 & 1 & 1 & 1 \\
\hline & $\mathrm{RF}$ & 65.65 & 50.26 & 27.19 & 35.29 & 63.96 & 32.18 & 16.70 & 21.99 & 58.38 & 18.43 & 14.42 & 16.18 \\
\hline \multirow{4}{*}{ ImageNet } & NB & 61.66 & 31.49 & 25.96 & 28.46 & 60.94 & 20.24 & 15.30 & 17.43 & 53.73 & 9.63 & 7.48 & 8.42 \\
\hline & K-NN & 76.02 & 50.14 & 47.36 & 48.71 & 74.80 & 32.19 & 33.63 & 32.89 & 68.32 & 20.22 & 21.46 & 20.82 \\
\hline & SVM & 76.69 & 55.66 & 46.89 & 50.90 & 1 & 1 & 1 & 1 & 1 & 1 & 1 & 1 \\
\hline & $\mathrm{RF}$ & 64.09 & 39.21 & 23.60 & 29.47 & 66.51 & 28.44 & 17.67 & 21.80 & 60.41 & 14.40 & 12.05 & 13.12 \\
\hline \multirow{4}{*}{$\begin{array}{c}\text { Par2vec } \\
+ \\
\text { ImageNet }\end{array}$} & NB & 64.3 & 28.2 & 24.5 & 26.22 & 63.41 & 16.79 & 13.25 & 14.81 & 55.68 & 8.40 & 6.04 & 7.03 \\
\hline & K-NN & 75.99 & 46.65 & 37.1 & 41.33 & 83.77 & 43.85 & 42.76 & 43.30 & 77.76 & 26.60 & 27.15 & 26.87 \\
\hline & SVM & 84.82 & 60.37 & 53.04 & 56.47 & 1 & 1 & 1 & 1 & 1 & 1 & 1 & 1 \\
\hline & $\mathrm{RF}$ & 69.98 & 46.66 & 23.27 & 31.05 & 70.44 & 28.67 & 27.55 & 28.10 & 65.21 & 17.94 & 15.16 & 16.43 \\
\hline
\end{tabular}

\section{Conclusion}

In this paper, we have proposed an approach that focuses on two tasks of the product integration pipeline, i.e., product matching and categorization. Our approach introduces the usage of unsupervised feature extraction for product data by using neural language modeling (word2vec and paragraph2vec) and deep learning models $(\mathrm{CNN})$. The highlights of this paper include: (i) word embeddings help the CRF model training significantly for "dirty" web data, (ii) text embeddings improve product categorization considerably, and (iii) image embeddings can be used as a weak signal for product matching and strong signal for product categorization. Moreover, we provide a thorough product specification fusion as a part of our use case of enriching product ads with semantic structured data.

Besides integrating products, our approach could be used for search query processing and information retrieval, which would improve the shopping experience for users [2].

\section{References}

[1] van Bezu, R., Borst, S., Rijkse, R., Verhagen, J., Vandic, D., Frasincar, F.: Multi-component similarity method for web product duplicate detection (2015)

[2] Bhattacharya, S., Gollapudi, S., Munagala, K.: Consideration set generation in commerce search. In: Proceedings of WWW. pp. 317-326. ACM (2011)
[3] DeNale, R., Weidenhamer, D.: Quarterly retail e-commerce sales 4th quarter 2015. US Census Bureau News (2015)

[4] Finkel, J.R., Grenager, T., Manning, C.: Incorporating non-local information into information extraction systems by gibbs sampling. In: Proceedings of the 43rd Annual Meeting on Association for Computational Linguistics. pp. 363-370 (2005)

[5] Ghani, R., Probst, K., Liu, Y., Krema, M., Fano, A.: Text mining for product attribute extraction. ACM SIGKDD Explorations Newsletter $8(1), 41-48$ (2006)

[6] Gopalakrishnan, V., Iyengar, S.P., Madaan, A., Rastogi, R., Sengamedu, S.: Matching product titles using web-based enrichment. In: 21st ACM international conference on Information and knowledge management. pp. 605-614 (2012)

[7] Grbovic, M., Radosavljevic, V., Djuric, N., Bhamidipati, N., Savla, J., Bhagwan, V., Sharp, D.: E-commerce in your inbox: Product recommendations at scale. In: Proceedings of the 21th ACM SIGKDD. pp. 18091818. ACM (2015)

[8] Isele, R., Bizer, C.: Learning linkage rules using genetic programming. In: Proceedings of the International Workshop on Ontology Matching. pp. 13-24 (2011)

[9] Kannan, A., Givoni, I.E., Agrawal, R., Fuxman, A.: Matching unstructured product offers to structured product specifications. In: 17th ACM SIGKDD (2011)

[10] Kannan, A., Talukdar, P.P., Rasiwasia, N., Ke, Q.: Improving product classification using images. In: Proceedings of the 2011 IEEE 11th International Conference on Data Mining. pp. 310-319. IEEE Computer Society (2011)

[11] Kiapour, M.H., Han, X., Lazebnik, S., Berg, A.C., Berg, T.L.: Where to buy it: Matching street clothing photos in online shops. In: 2015 IEEE International Conference on Computer Vision (ICCV). pp. 3343-3351 (Dec 2015)

[12] Köpcke, H., Thor, A., Thomas, S., Rahm, E.: Tailoring entity resolution for matching product offers. In: Proceedings of the 15th International Conference on Extending Database Technology. pp. 545-550. ACM (2012)

[13] Krizhevsky, A., Sutskever, I., Hinton, G.E.: Imagenet classification with deep convolutional neural networks. In: Advances in neural information processing systems. pp. 1097-1105 (2012)

[14] Le, Q.V., Mikolov, T.: Distributed representations of sentences and documents. arXiv preprint arXiv:1405.4053 (2014)

[15] LeCun, Y., Boser, B., Denker, J.S., Henderson, D., Howard, R.E., Hubbard, W., Jackel, L.D.: Backpropagation applied to handwritten zip code 
recognition. Neural computation 1(4), 541-551 (1989)

[16] Londhe, N., Gopalakrishnan, V., Zhang, A., Ngo, H.Q., Srihari, R.: Matching titles with cross title web-search enrichment and community detection. Proceedings of the VLDB Endowment 7(12), 1167-1178 (2014)

[17] McAuley, J., Targett, C., Shi, Q., van den Hengel, A.: Image-based recommendations on styles and substitutes. In: Proceedings of the 38th International ACM SIGIR Conference. pp. 43-52. ACM (2015)

[18] Melli, G.: Shallow semantic parsing of product offering titles (for better automatic hyperlink insertion). In: Proceedings of the 20th ACM SIGKDD international conference on Knowledge discovery and data mining. pp. 1670-1678. ACM (2014)

[19] Meusel, R., Petrovski, P., Bizer, C.: The webdatacommons microdata, rdfa and microformat dataset series. In: The Semantic Web-ISWC, pp. 277-292 (2014)

[20] Meusel, R., Primpeli, A., Meilicke, C., Paulheim, H., Bizer, C.: Exploiting microdata annotations to consistently categorize product offers at web scale. In: Proceedings of EC-Web. Valencia, Spain (2015)

[21] Mikolov, T., Chen, K., Corrado, G., Dean, J.: Efficient estimation of word representations in vector space. arXiv preprint arXiv:1301.3781 (2013)

[22] Mikolov, T., Sutskever, I., Chen, K., Corrado, G.S., Dean, J.: Distributed representations of words and phrases and their compositionality. In: Advances in neural information processing systems. pp. 3111-3119 (2013)

[23] Nguyen, H., Fuxman, A., Paparizos, S., Freire, J., Agrawal, R.: Synthe- sizing products for online catalogs. Proceedings of the VLDB Endowment 4(7), 409-418 (2011)

[24] Petrovski, P., Bryl, V., Bizer, C.: Integrating product data from websites offering microdata markup. In: Proceedings of the companion publication of the 23rd international conference on World wide web companion. pp. 1299-1304 (2014)

[25] Petrovski, P., Bryl, V., Bizer, C.: Learning regular expressions for the extraction of product attributes from e-commerce microdata (2014)

[26] Ristoski, P., Mika, P.: Enriching product ads with metadata from html annotations. In: Proceedings of the 13th Extended Semantic Web Conference. (To Appear) (2016)

[27] de Souza, J.G., Federico, M., Sawaf, H.: Mt quality estimation for ecommerce data

[28] Vandic, D., Van Dam, J.W., Frasincar, F.: Faceted product search powered by the semantic web. Decision Support Systems 53(3), 425-437 (2012)

[29] Wang, M., Manning, C.D.: Effect of non-linear deep architecture in sequence labeling. In: IJCNLP. pp. 1285-1291 (2013)

[30] Zhao, W.X., Li, S., He, Y., Chang, E., Wen, J.R., Li, X.: Connecting social media to e-commerce: Cold-start product recommendation on microblogs. IEEE Transactions on Knowledge and Data Engineering PP(99), 1-1 (2015) 